\title{
Spinal Cord Gray Matter Atrophy in Amyotrophic Lateral Sclerosis
}

\author{
(D) M.-Ê. Paquin, (D) M.M. El Mendili, (D) C. Gros, (D) S.M. Dupont, DJ. Cohen-Adad, and (DP.-F. Pradat
}

\begin{abstract}
BACKGROUND AND PURPOSE: There is an emerging need for biomarkers to better categorize clinical phenotypes and predict progression in amyotrophic lateral sclerosis. This study aimed to quantify cervical spinal gray matter atrophy in amyotrophic lateral sclerosis and investigate its association with clinical disability at baseline and after 1 year.
\end{abstract}

MATERIALS AND METHODS: Twenty-nine patients with amyotrophic lateral sclerosis and 22 healthy controls were scanned with 3T MR imaging. Standard functional scale was recorded at the time of MR imaging and after 1 year. MR imaging data were processed automatically to measure the spinal cord, gray matter, and white matter cross-sectional areas. A statistical analysis assessed the difference in crosssectional areas between patients with amyotrophic lateral sclerosis and controls, correlations between spinal cord and gray matter atrophy to clinical disability at baseline and at 1 year, and prediction of clinical disability at 1 year.

RESULTS: Gray matter atrophy was more sensitive to discriminate patients with amyotrophic lateral sclerosis from controls $(P=.004)$ compared with spinal cord atrophy $(P=.02)$. Gray matter and spinal cord cross-sectional areas showed good correlations with clinical scores at baseline $(R=0.56$ for gray matter and $R=0.55$ for spinal cord; $P<.01)$. Prediction at 1 year with clinical scores $\left(R^{2}=0.54\right)$ was improved when including a combination of gray matter and white matter cross-sectional areas $\left(R^{2}=0.74\right)$.

CONCLUSIONS: Although improvements over spinal cord cross-sectional areas were modest, this study suggests the potential use of gray matter cross-sectional areas as an MR imaging structural biomarker to monitor the evolution of amyotrophic lateral sclerosis.

ABBREVIATIONS: ALS = amyotrophic lateral sclerosis; ALSFRS-R = arm-revised ALS Functional Rating Scale; CSA = cross-sectional area; GMCSA = gray matter cross-sectional area; $\mathrm{SC}=$ spinal cord; $\mathrm{SCCSA}=$ spinal cord cross-sectional area

A lthough amyotrophic lateral sclerosis (ALS) remains a relatively rare disease (median incidence rate was 2.08 per 100,000 in Europe for $2010,{ }^{1}$ the loss of autonomy, short survival rate (median survival from onset, 23-52 months), ${ }^{1,2}$ and lack of proper treatment motivate the development of robust biomarkers to better categorize clinical phenotype and improve prognosis. ${ }^{3,4} \mathrm{Com}-$

Received March 30, 2017; accepted after revision August 17.

From the Faculté de Médecine (M.-Ê.P.) and Functional Neuroimaging Unit, CRIUGM (J.C.-A.), Université de Montréal, Montreal, Quebec, Canada; NeuroPoly Lab, Institute of Biomedical Engineering, Polytechnique Montreal (M.-Ê.P., C.G., S.M.D., J.C.-A.), Montreal, Quebec, Canada; Sorbonne Universités (M.M.E.M., P.-F.P.) UPMC Univ Paris 06, CNRS, INSERM, Laboratoire d'Imagerie Biomédicale, Paris, France; Department of Neurology (M.M.E.M.), Icahn School of Medicine, Mount Sinai, New York, New York; and Département des Maladies du Système Nerveux (P.-F.P.), Centre Référent Maladie Rare SLA, Hôpital de la Pitié-Salpêtrière, Paris, France.

This work was supported by the Canada Research Chair in Quantitative Magnetic Resonance Imaging (J.C.-A.), the Canadian Institute of Health Research (CIHR FDN143263), the Canada Foundation for Innovation (32454), the Fonds de Recherche du Québec - Santé (28826), the Fonds de Recherche du Québec - Nature et Technologies (2015-PR-182754), the Natural Sciences and Engineering Research Council of Canada (435897-2013), the Quebec Biolmaging Network and the Association Française contre les Myopathies, the Institut pour la Recherche sur la Moelle épinière et l'Encéphale, and the "Investissements d'avenir" (ANR-10-IAIHU-06). mon clinical manifestations include muscle weakness or clumsiness, atrophy, cramps, fasciculations, dysphagia, dysarthria, and respiratory symptoms such as dyspnea, orthopnea, and respiratory failure. ${ }^{2}$ Because the clinical presentation and progression rate are highly heterogeneous, ${ }^{5}$ it remains challenging to identify the true biologic effects of drug testing in clinical trials. Exploring new processing methods and hypotheses would promote a greater understanding of the physiopathologic processes. ${ }^{6}$ In particular, imaging biomarkers of the spinal cord (SC) can potentially provide a relevant measure of the degeneration of lower motor neurons. ${ }^{3,4}$

A recent study showed that spinal cord cross-sectional area (SCCSA) measured with MR imaging improves prediction of the arm-revised ALS Functional Rating Scale (ALSFRS-R) subscore at 1 year. ${ }^{3}$ Another study ${ }^{7}$ established a strong link between SCCSA and the degeneration of lower motor neurons. The main limita-

Please address correspondence to Julien Cohen-Adad, PhD, Département Genie Electrique, L5610, Ecole Polytechnique, 2900 Edouard-Montpetit Blvd, Montreal, Quebec H3T 1J4, Canada; e-mail: jcohen@polymtl.ca

- Indicates open access to non-subscribers at www.ajnr.org

http://dx.doi.org/10.3174/ajnr.A5427 
tion of these studies is that they looked at cord atrophy, hindering the separation of the specific contribution of GM and WM atrophy. Recent advances in analysis tools for segmenting the SC GM ${ }^{8}$ now permit such exploration.

The objective of the present study was to quantify spinal GM atrophy in ALS and to investigate its association with clinical disability at baseline and after 1 year.

\section{MATERIALS AND METHODS \\ Patients}

Twenty-nine patients with ALS and 22 age-matched controls were recruited at the ALS Center of the Pitié-Salpêtrière Hospital in Paris, France. Patients with ALS were diagnosed with probable $(n=20)$, laboratory probable or definite $(n=9)$ ALS according to the El Escorial criteria. ${ }^{9}$ Exclusion criteria included important acute and chronic medical conditions interfering with the clinical evaluation, significant psychiatric or neurologic history (other than ALS for patients), and standard contraindications to MR imaging. Controls had no known neurologic disorder and no family history of neurologic diseases, and they were recruited to identify MR imaging markers in the SC that distinguish them from patients with ALS. The study was reviewed by the local ethics committee board, and written informed consent was obtained from each participant.

The following clinical assessments were conducted on patients with ALS: ALSFRS-R (total score and arm subscore [arm subscore included evaluation of handwriting, cutting food, and handling kitchen utensils]), ${ }^{10}$ manual muscle testing (7 proximal and distal muscles of the arm were tested) by using the Medical Research

Table 1: Demographic data for the 25 patients with ALS and the 22 healthy controls

\begin{tabular}{lccc} 
& & \multicolumn{2}{c}{$\begin{array}{c}\text { Patients with } \\
\text { ALS }\end{array}$} \\
\cline { 3 - 4 } & & \multicolumn{2}{c}{$\begin{array}{c}\text { 1-Year } \\
\text { Follow-Up } \\
\text { Subgroup }\end{array}$} \\
\cline { 3 - 4 } \multicolumn{1}{c}{ Characteristics } & Controls & At Baseline & Subgroun \\
Number & 22 & 25 & 19 \\
Sex & $11 \mathrm{~F} / 11 \mathrm{M}$ & $6 \mathrm{~F} / 19 \mathrm{M}$ & $3 \mathrm{~F} / 16 \mathrm{M}$ \\
Age at baseline, $y \mathrm{yr} \pm \mathrm{SD}$ & $50.9 \pm 13.0$ & $53.3 \pm 10.1$ & $52.8 \pm 9.2$ \\
\hline
\end{tabular}

Note:-F indicates female; $M$, male.
Council score. The progression rate at baseline of the ALSFRS-R score was defined as $\Delta$ by the following equation:

$$
\Delta=\left(\operatorname{ALSFRS}_{\mathrm{Max}}-\mathrm{ALSFRS}_{\mathrm{MRI}}\right) / \tau
$$

where ALSFRS $_{\text {Max }}=48$, corresponding to the maximum ALSFRS-R score (ie, that of a healthy control); ALSFRS $_{\text {MRI }}$ is the ALSFRS-R score at the time of MR imaging; and $\tau$ is the delay (in months) between the first symptoms and the time of MR imaging. The progression rate $\Delta$ thus corresponds to the "speed" of the progression of the disease at the time of MR imaging (ie, baseline).

ALSFRS-R and manual muscle testing were performed on the same day as the MR imaging acquisition. Clinical evaluation was performed by an experienced neurologist specialized in ALS (P.-F.P., 15 years' experience).

One patient with ALS was excluded from the study because the MR imaging acquisition was interrupted for medical reasons, and 3 patients were excluded because of excessive motion during acquisition. The total number of patients used in this study was 25 . Table 1 lists demographic data, Table 2 lists clinical data commonly used for prognosis, and Table 3 lists clinical scores at baseline and 1 year after.

\section{MR Imaging Acquisition}

Data were acquired on a 3T MR imaging system (Tim Trio; Siemens, Erlangen, Germany) by using the product 12-channel head, 4-channel neck, and the 3 most rostral elements of a spine coil for signal reception.

A 3D T2-weighted fast spin-echo sequence (sampling perfection with application-optimized contrasts by using different flip angle evolution, or SPACE) was acquired as an anatomic image for subsequent registration to a common template (see "Data Processing" section). Parameters were: TR, $1500 \mathrm{~ms}$; TE, $120 \mathrm{~ms} ; 52$ sections; field of view, $280 \times 280 \mathrm{~mm}^{2}$; voxel size, $0.9 \times 0.9 \times 0.9 \mathrm{~mm}^{3}$; acceleration factor, $R=2$; and acquisition time, 3 minutes.

An axial 2D T2*-weighted multiecho gradient-echo sequence (multiecho data image combination, or MEDIC; Siemens proprietary sequence) was acquired for GM cross-sectional area (GMCSA) measurements thanks to the good white-to-gray matter contrast available in this type of sequence. Parameters were: TR, $470 \mathrm{~ms}$; average TE, $17 \mathrm{~ms}$; 23 sections; field of view, $180 \times$

Table 2: Clinical data commonly used as prognostic factors for the 25 patients with ALS

\begin{tabular}{lcc} 
& & Patients with ALS \\
\cline { 2 - 3 } \multicolumn{1}{c}{ Clinical Data } & Baseline & 1-Year Follow-Up Subgroup \\
\hline Body mass index at MRI ( \pm SD) & $23.7 \pm 2.7$ & $24.0 \pm 2.7$ \\
Familial transmission & 2 SODI mutated & 1 SODI mutated \\
Site of onset & 1 bulbar; 18 upper; 6 lower & 1 bulbar; 12 upper; 6 lower \\
Delay from first symptom to diagnosis, mo \pm SD & $9.9 \pm 5.6$ & $10.7 \pm 6.0$ \\
\hline
\end{tabular}

Note:-SOD1 indicates SuperOxide Dismutase 1 gene.

Table 3: Clinical scores ${ }^{a}$

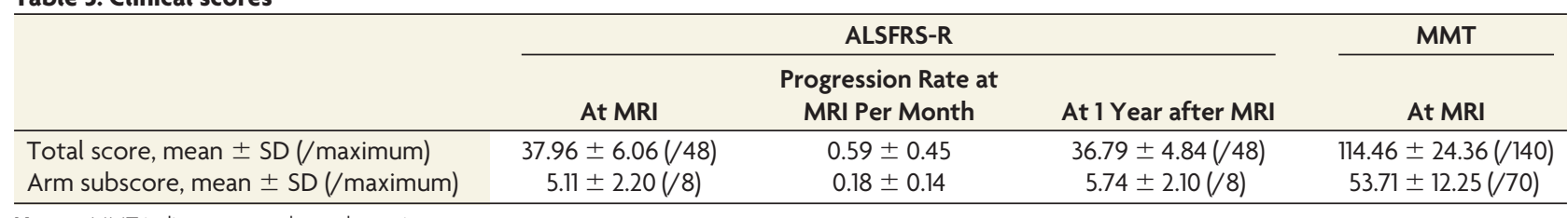

Note:-MMT indicates manual muscle testing.

${ }^{a}$ Clinical scores are presented both in total (top row) and for the upper limbs only (arm subscore, bottom row). Presented clinical scores are, from left to right: the revised ALSFRS-R at the time of MRI, ALSFRS-R progression rate at MRI, ALSFRS-R at 1 year after MRI, and MMT at MRI. The progression rate represents the decline of ALSFRS-R score per month between the first onset of symptoms and the MRI. 


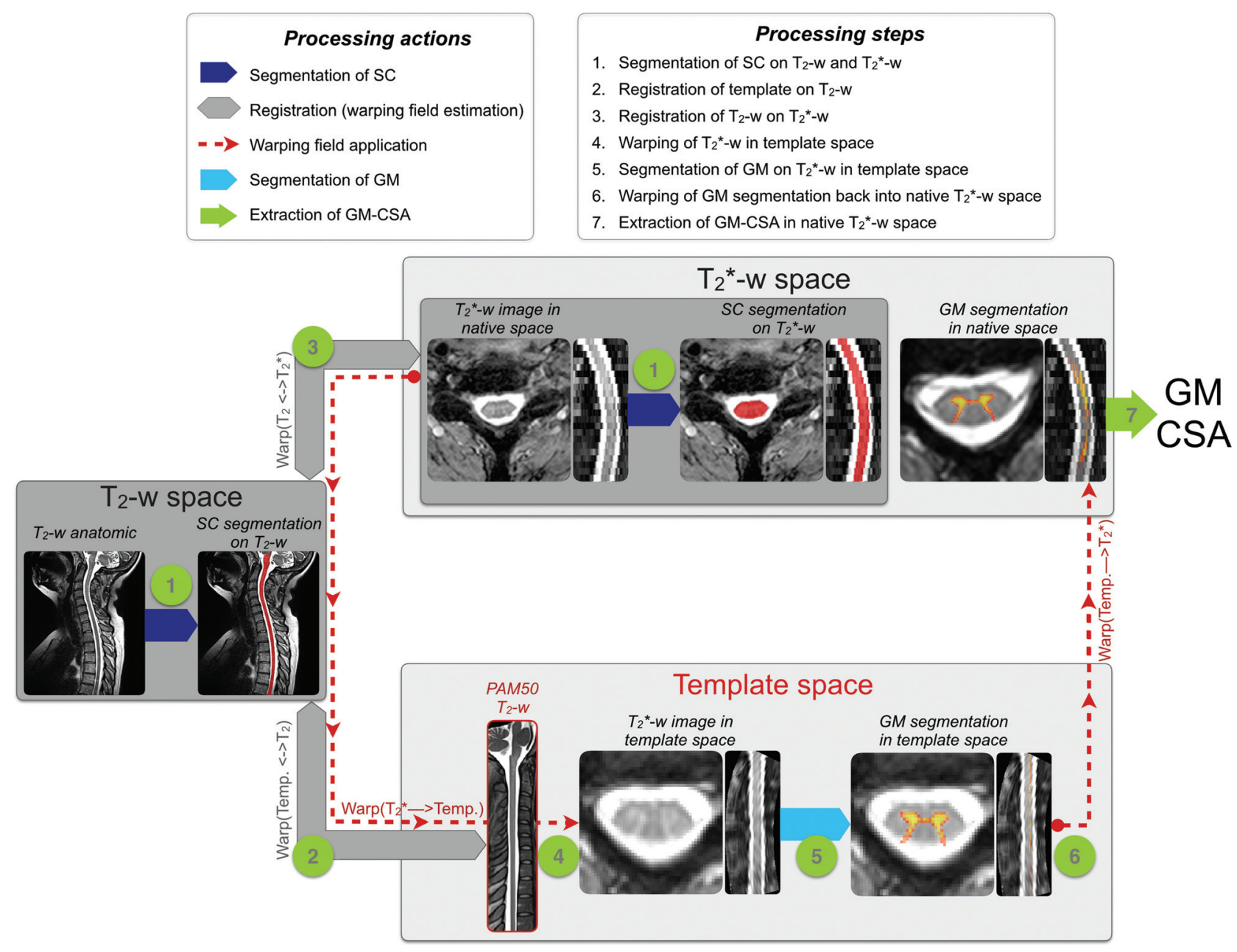

FIG 1. Processing pipeline for the GM segmentation and computation of the GMCSA.

$180 \mathrm{~mm}^{2}$; voxel size, $0.7 \times 0.7 \times 3 \mathrm{~mm}^{3}$; acceleration factor, $R=$ 2 ; acquisition time, 3 minutes; and intersection gap, $1.5 \mathrm{~mm}$.

Acquisition parameters and .edx protocols can be downloaded from the Open Science Framework public repository (https://osf.io/9xkxx/).

\section{Data Processing}

All data were processed by using the open-source software Spinal Cord Toolbox v3.0. ${ }^{11}$ All processing commands are available at the Open Science Framework public repository. In brief, T2-weighted and $\mathrm{T} 2{ }^{\star}$-weighted images were automatically registered to a common template image, which was generated in a previous study by averaging spinal cord MRIs from 50 adult subjects. ${ }^{12,13}$ The T2weighted image was used to precisely identify vertebral levels, and the T2* -weighted image was used to automatically measure the GMCSA thanks to its good white-to-gray matter contrast. Figure 1 illustrates the processing pipeline, which can be broken down into 7 steps:

1) The spinal cord was automatically segmented by using "PropSeg" 14 on T2-weighted and T2*-weighted images. Note that the segmentation was slightly manually corrected when needed.

2) The PAM50 template ${ }^{15}$ was registered to the T2-weighted anatomic data by using 3 consecutive steps: i) a section-wise rigid transformation (translation and rotation) based on the center of mass of the segmentations; ii) a nonrigid regularized registration by using the BSplineSyn algorithm ${ }^{16}$ based on cord segmentations; and iii) a nonrigid registration by using the SyN algorithm ${ }^{17}$ on the images (as implemented in Advanced Normalization Tools $\left.{ }^{18}\right)$.

3) The T2-weighted template registered into the T2-weighted anatomic space was then registered on the $\mathrm{T} 2{ }^{\star}$-weighted image by using 3 consecutive steps: i) a section-wise regularized rigid registration ${ }^{19}$ based on cord segmentations; ii) a nonrigid regularized registration by using the BSplineSyn algorithm on cord segmentations; and iii) a nonrigid registration by using the SyN algorithm on the images.

4) Both registrations (template to T2-weighted and T2weighted space to $\mathrm{T} 2{ }^{\star}$-weighted) output forward- and backwardwarping fields. Concatenating the 2 forward-warping fields allows for warping the template (and all its elements) into the $\mathrm{T} 2{ }^{*}$ weighted space (WARP[template to $\left.\mathrm{T} 2^{\star}\right]$ ), and concatenating all the backwards-warping fields allows for warping the $\mathrm{T} 2^{\star}$ weighted image into the template space (WARP $\left[\mathrm{T}^{*}\right.$ to template]). Here, we took advantage of the second concatenation (WARP $\left[\mathrm{T}^{*}\right.$ to template]) to warp the $\mathrm{T} 2^{\star}$-weighted image and the automatic SC segmentation into the template space.

5) The GM was automatically segmented on the $\mathrm{T} 2^{\star}$-weighted image in the template space. The GM segmentation was performed by using the multiatlas-based segmentation method in- 


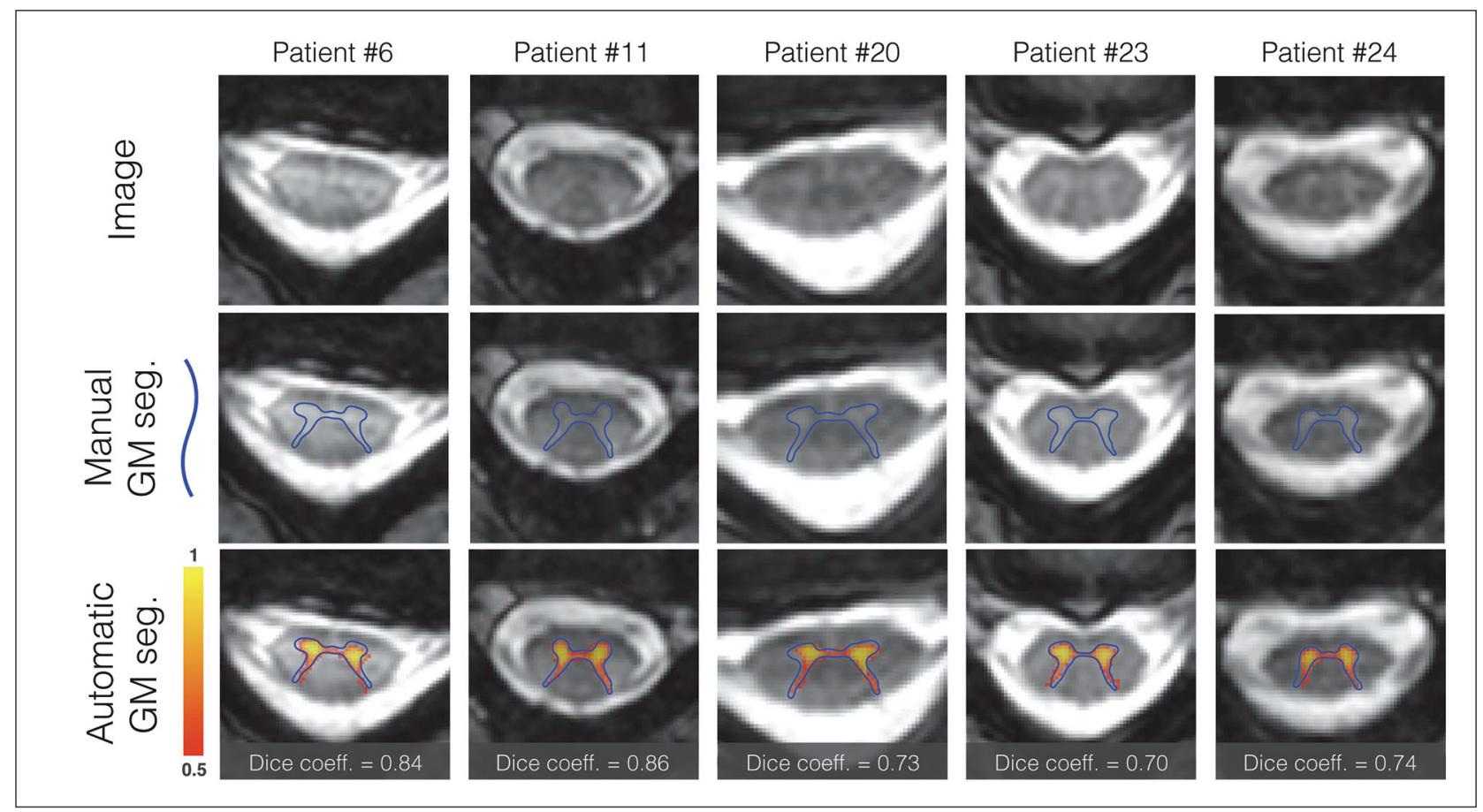

FIG 2. GM automatic segmentation and manual delineation patients with ALS. Manual delineation of the GM is displayed with the blue line, automatic probabilistic segmentation is shown in red-to-yellow. Dice coefficient comparing the automatic and manual segmentation is shown on the bottom line.

cluded in the Spinal Cord Toolbox. ${ }^{8}$ The automatic GM segmentation was visually assessed for all patients. A more thorough quantitative validation has been conducted in a previous study. ${ }^{8}$

6) The GM segmentation was warped back into the $T 2^{*}$ weighted space by using the forward-warping field (WARP[template to $\left.\mathrm{T}^{\star}\right]$ ).

7) The SCCSA and GMCSA were computed in the T2*weighted space. The area computed for each section was corrected for the curvature of the spine by using the angle of the section with the SC centerline. Note that the cross-sectional area (CSA) was not normalized across subjects, as further discussed in the "Methodologic Considerations" section.

\section{Statistical Analysis}

Differences between Patients and Controls. All statistical analyses were performed with Python 2.7. A 1-sample KolmogorovSmirnov test was used to confirm that SCCSA and GMCSA followed a Gaussian distribution within the patients and controls groups. Consequently, a 2-sample Student $t$ test assessed potential CSA differences between the 2 groups.

Correlation between GM Atrophy and Clinical Disability at MR Imaging (Baseline) and at 1 Year After. The association between spinal cord atrophy and clinical disability at baseline and at 1 year was investigated. The hypothesis was that atrophy of the cervical GM measured in MR imaging is associated with clinical outcome. At baseline, Pearson correlation coefficients between CSA of GM and SC and ALSFRS-R/manual muscle testing scores were computed. Similarly, Pearson correlation coefficients were computed between GMCSA and ALSFRS-R score and between SCCSA and ALSFRS-R score at 1 year after baseline with the 19 patients with
ALS who were available to follow-up (referred to as the 1-year cohort). GMCSA and SCCSA values were averaged across the C4-C6 vertebral levels to be more specific with the arm subscore, which is associated with shoulder abduction (myotome C5), elbow flexion (myotome C6), and wrist extension (myotome C7).

Prediction of Clinical Disability at 1 Year After Baseline. The prediction of clinical disability was performed on the 1-year cohort. Regression trees ${ }^{20}$ were used to evaluate the potential of MR imaging biomarkers to predict the total ALSFRS-R score at 1 year after MR imaging. Regression trees as used here are a supervised learning method aimed at predicting the ALSFRS-R score at 1 year by learning simple decision rules inferred from clinical predictors (sex, age, body mass index, site of onset, delay between first symptom and diagnosis, ALSFRS progression rate at baseline [see equation 1], and ALSFRS-R at baseline) and MR imaging measures. Several models were tested with 1) clinical predictors only; 2) clinical predictors and SCCSA; and 3) clinical predictors, GMCSA, and the ratio of WM CSA to GMCSA. MR imaging biomarkers were progressively added to the clinical predictors to test the impact of each MR imaging measure. The hypothesis was that at 1 year, the prediction score of disability would be higher when adding MR imaging measures at baseline to clinical predictors. Here, WM CSA was used instead of SCCSA to avoid colinearity between predictors (because SCCSA $=$ WM CSA + GMCSA). As in the previous subsection, GMCSA and WM CSA values were averaged across the C4-C6 vertebral levels.

A leave-one-out cross-validation was performed to evaluate how each prediction model will generalize to a new dataset. A 


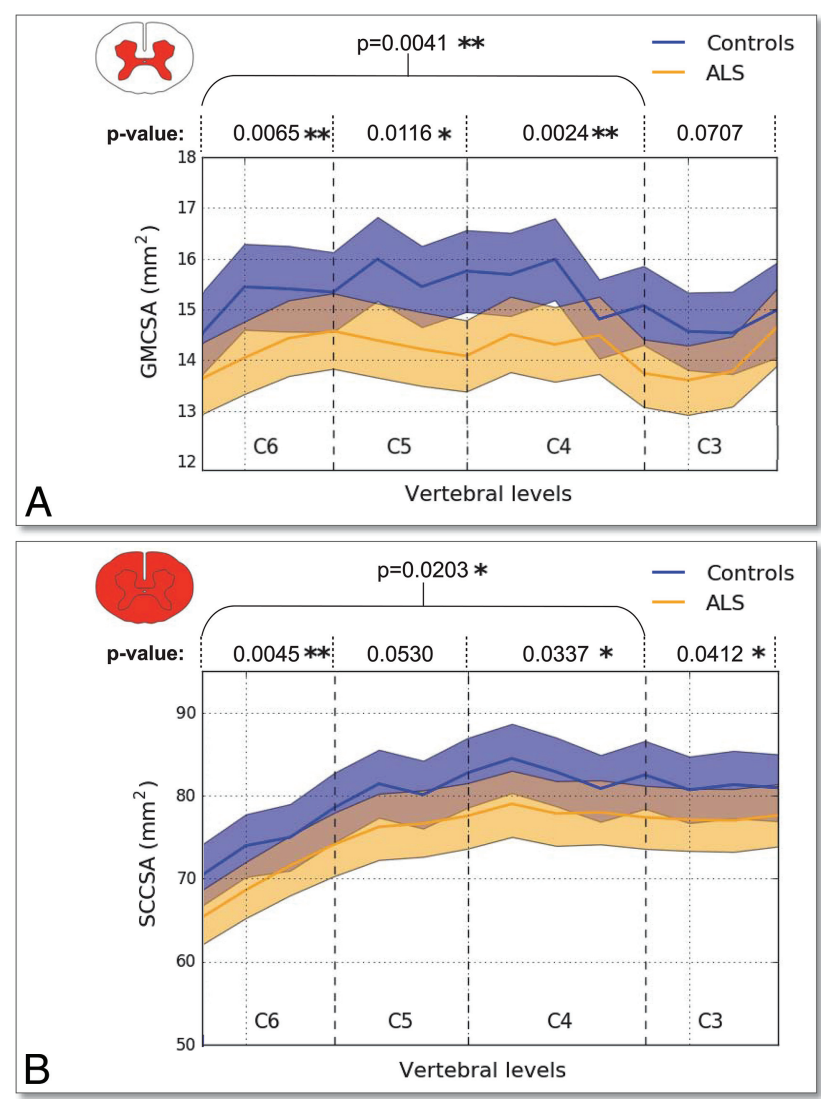

FIG 3. GMCSA and SCCSA measured on controls and patients with $A L S$ between the C6-C3 vertebral levels. GMCSA $(A)$ and SCCSA $(B)$ averaged within group and plot against the cervical $S C$ axis. Overall, a stronger intergroup difference can be observed for GMCSA. Asterisk $(P \leq .05)$ and double asterisk $(P \leq .01)$ at specific vertebral levels indicate significant differences between patients with ALS and controls according to Student $t$ test $P$ values representing control-to-patient differences in GMCSA and SCCSA for each cervical level between C 6 and C 3 and across levels.

patient was randomly discarded from the list of patients, and a new prediction model was created from the remaining $(n-1)$ patients. From this model, we computed the prediction error, which is defined as the difference between the true and the predicted value of the ALSFRS-R score. This procedure was run 25 times, and the distribution of error was reported.

\section{RESULTS}

\section{Data Processing}

The proposed processing pipeline was fully automatic. The results have been visually inspected by an experimented rater, and a manual correction was only needed for the SC segmentation of 8 patients with ALS out of the 47 processed subjects ( 22 controls and 25 patients). The correction took approximately 1 minute per subject and consisted of a slight manual adjustment of the SC segmentation on $\mathrm{T}^{*}$-weighted images. The accuracy of the GM automatic segmentation has been validated in 5 randomly selected patients with ALS, with a Dice coefficient of 0.708 . Figure 2 shows a visual illustration of the GM segmentation.

\section{Differences between Patients and Controls}

Figure 3 shows the average $( \pm$ SD) of GMCSA (Fig 3A) and SCCSA (Fig 3B) in controls (blue) and patients with ALS (orange) across the SC cervical axis. Qualitatively, a larger difference between patients and controls is observed for GMCSA compared with SCCSA (7.2\% difference for GMCSA versus 3.6\% difference for SCCSA averaged between the C4-C6 vertebral levels, $t$ test $P$ values for each vertebral level are shown in Fig 3). Figure 4 shows a boxplot distribution of GMCSA and SCCSA averaged between the C4-C6 vertebral levels.

\section{Correlation between GM Atrophy and Clinical Disability at MR Imaging (Baseline) and at 1 Year After}

A correlation study was performed between clinical disability (at baseline, then at 1 year after MR imaging) and MR imaging biomarkers of grouped vertebral levels (C4-C6) (Table 4). GMCSA and SCCSA measures revealed significant correlations with the ALSFRS-R subscore at baseline and at 1 year after, suggesting an association between cervical GM atrophy and clinical disability of the upper limbs at baseline as well as after 1 year. For the manual muscle testing arm subscore at baseline, significant correlations were only found for GMCSA, although $P$ values between GMCSA and SCCSA were very close (.049 versus .054 [Table 4]).

\section{Prediction of Clinical Disability at 1 Year After Baseline}

The purpose of this analysis was to investigate the benefits of adding MR imaging biomarkers to clinical predictors in a generalizable model of clinical disability prediction.

Table 5 shows the results of regression trees between total ALSFRS-R score at 1 year after baseline and several predictors frequently used in practice, with or without MR imaging biomarkers. Figure 5 shows the distribution of the prediction error for the 3 prediction models based on a leave-one-out cross-validation, suggesting that the prediction model including MR imaging biomarkers results in a more accurate score prediction. The model including all the proposed MR imaging biomarkers predicted the ALSFRS-R score with an average error of $1.63 \pm 8.42$ versus $2.05 \pm 12.97$ with clinical predictors only.

\section{DISCUSSION}

This study focused on using the CSA of the cervical SC GM in patients with ALS for 1) discriminating between patients with ALS and controls; 2) correlating CSA with clinical scores (ALSFRS-R and manual muscle testing); and 3) predicting clinical score (ALSFRS-R) at 1-year follow-up. The following subsections will compare our results with the previous literature and discuss their limitations and relevance for clinical implications.

\section{GM Atrophy Detected in Patients with ALS}

SCCSA exhibited significant differences between patients with ALS and healthy controls, confirming the results obtained by another study. ${ }^{3}$ More interestingly, GMCSA showed larger differences between the 2 groups ( $P=.004$ for GMCSA versus .02 for SCCSA at the C4-C6 vertebral levels), suggesting that GMCSA is a more sensitive marker of atrophy in ALS and that cord atrophy in ALS is predominantly driven by lower motor neuron degeneration. Although WM is expected to degenerate as a secondary effect of upper motor neuron degeneration, gliosis and myelin debris forming in the SC WM might somewhat lower the sensitivity of global cord atrophy. ${ }^{21,22}$ 

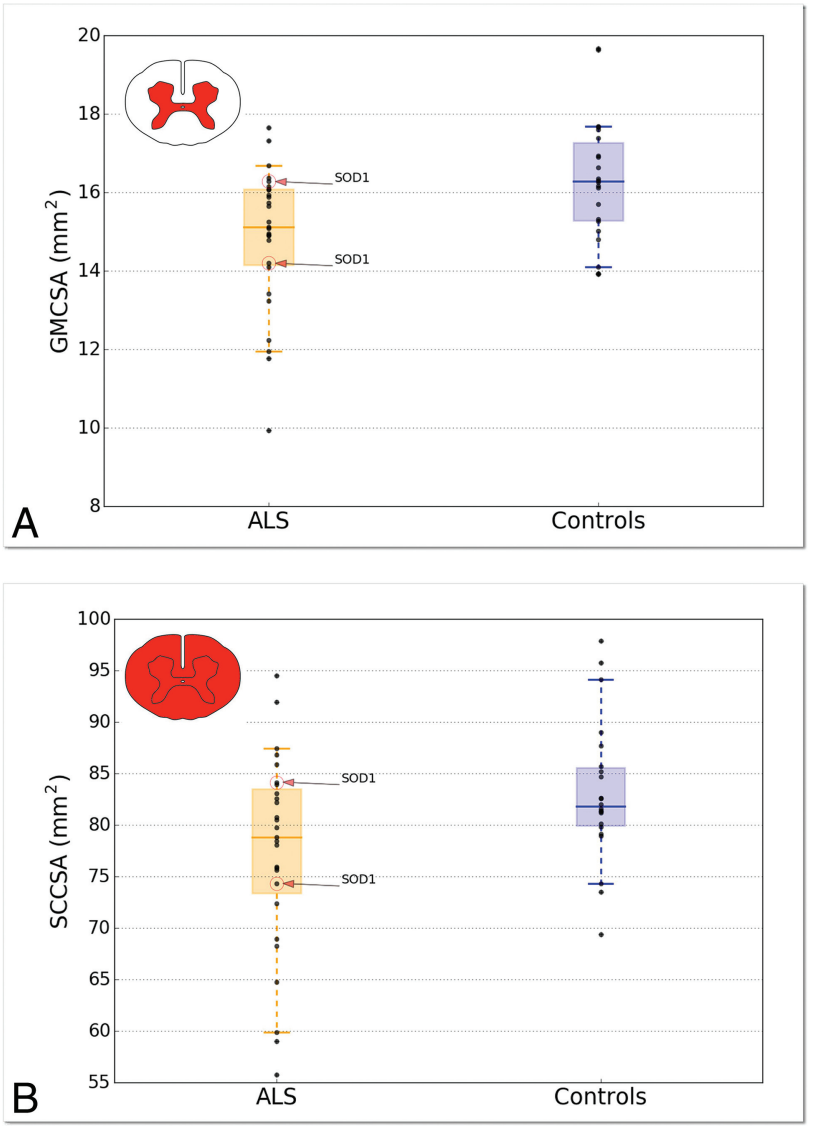

FIG 4. Boxplot distribution of GMCSA $(A)$ and SCCSA $(B)$ averaged between the $\mathrm{C} 4-\mathrm{C} 6$ vertebral levels. Each dark point represents an individual value. The median is represented as a thick horizontal line and the interquartile range as a light rectangle. The horizontal bar at both extremities of the whiskers represent the 5th and 95th percentiles. The 2 patients presenting the SODl gene are identified in the plot.

In addition, the control-to-patient difference was larger at the C4-C6 vertebral level region (Fig 3), which is the site of large pools of motor neurons. Larger GM atrophy at the C4-C6 vertebral levels could be explained by a larger absolute number of at-

Table 4: Correlation coefficients between CSA (GM and SC) and clinical scores at baseline and at 1 year ${ }^{\mathrm{a}}$

\begin{tabular}{|c|c|c|c|}
\hline \multirow[b]{2}{*}{ Predictors } & \multicolumn{2}{|c|}{ At Baseline } & \multirow{2}{*}{$\begin{array}{l}\text { At } 1 \text { Year } \\
\text { ALSFRS-R } \\
\text { Subscore }\end{array}$} \\
\hline & $\begin{array}{l}\text { ALSFRS-R } \\
\text { Subscore }\end{array}$ & $\begin{array}{l}\text { MMT Arm } \\
\text { Subscore }\end{array}$ & \\
\hline GMCSA & $\begin{array}{l}R=0.56 \\
P=.004^{b}\end{array}$ & $\begin{array}{l}R=0.40 \\
P=.049^{a}\end{array}$ & $\begin{array}{l}R=0.48 \\
P=.035^{c}\end{array}$ \\
\hline SCCSA & $\begin{array}{l}R=0.55 \\
P=.005^{b}\end{array}$ & $\begin{array}{l}R=0.40 \\
P=.054\end{array}$ & $\begin{array}{l}R=0.54 \\
P=.017^{c}\end{array}$ \\
\hline
\end{tabular}

Note:-MMT indicates manual muscle testing.

${ }^{a}$ CSA was averaged across the C4-C6 vertebral levels. Clinical scores included: 1) ALSFRS-R at baseline; 2) MMT subscores at baseline; and 3) ALSFRS-R subscore at 1 year.

b Significant $(P \leq .01)$.

c Significant $(P \leq .05)$.

rophied motor neurons at this level and/or by a higher sensitivity of the MR imaging-based GMCSA measure at this level because of the increased size of the structure that facilitates delineation of the GM interface. Further studies covering a larger portion of the spinal cord would shed light on these possible explanations.
From a clinical perspective, having SCCSA and GMCSA highly correlated raises the question of the relevance of measuring GMCSA in patients with ALS as a diagnostic measure, especially given that it is more difficult to reliably measure GMCSA from standard clinical scans because of the need for high axial resolution and sufficient GM/WM contrast-to-noise. Nevertheless, having access to a more specific assessment of GM atrophy still has potential for monitoring the efficiency of new drugs, though this has not been tested here.

\section{GM Atrophy Correlates with Clinical Disability at MR Imaging and 1 Year After}

As shown in Table 4, GMCSA seems to be a relevant biomarker of clinical disability at vertebral levels C4-C6 (ALSFRS-R and manual muscle testing arm at baseline, $P=.004$ and $P=.05$, respectively; ALSFRS-R at 1 year, $P=.03$ ). SCCSA also reflected clinical disability as previously shown, ${ }^{3}$ though with lower sensitivity than GMCSA. A previous study ${ }^{7}$ established a strong link between SCCSA and lower motor neuron degeneration by relating motorevoked potential amplitude of the adductor digiti minimi and deltoid, respectively, with cord atrophy at spinal levels C8 and C5 (here, it was not possible to study the C8 spinal level; see "Limitations" section). However, combining advanced image processing and better MR imaging pulse sequences is promising to investigate the effect of GM atrophy on muscle-specific deficits in the low thoracic and lumbar cord. ${ }^{23}$ The ability to isolate lower motor neuron from upper motor neuron contribution in ALS might provide additional information for understanding the pathogenesis of the disease.

\section{Prediction of Clinical Disability at 1 Year After Baseline}

A significant association was found between GMCSA (measured at baseline) and the ALSFRS-R score at 1 year after MR imaging acquisition $(P=.03)$. However, it was not possible to perfectly fit a regression model to predict clinical disability at 1 year by using clinical biomarkers and MR imaging measures (CSA), which is challenging regarding the heterogeneity of the present ALS cohort in terms of clinical score and site of onset. The best prediction score was obtained by combining MR imaging measures (GM, $\mathrm{WM}$, and ratio of WM CSA to GMCSA). Therefore, this suggests that the association of MR imaging measures could be helpful to predict the evolution of clinical disability for patients with ALS ( $R^{2}=0.54$ without versus $R^{2}=0.74$ with MR imaging measures). Evaluated with a leave-one-out cross-validation, prediction accuracy and generalization were improved by including MR imaging biomarkers in the prediction model (error $=1.63 \pm 8.42$ with versus $2.05 \pm 12.97$ without MR imaging measures). More complex models such as deep learning would be a potential alternative to achieve a more specific prediction. ${ }^{24}$

\section{Methodologic Considerations}

Classical Bias in ALS Studies. Because patients with ALS with heavy respiratory symptoms could not be recruited for the study, a lack of external validity needs to be pointed out. By recruiting subjects in a less drastic state (from a medical standpoint), a certain selection bias takes place that limits generalization to the whole ALS population and affects the power of the study. Moreover, the recruited population included mostly 
Table 5: Results of regression tree predictions to make prediction of total ALSFRS-R score at 1 year after MRI with several clinical and MRI predictors ${ }^{\mathrm{a}}$

\begin{tabular}{lcc}
\multicolumn{1}{c}{ Predictors } & $\begin{array}{c}\text { Coefficient of } \\
\text { Determination } \\
\left.\boldsymbol{R}^{2} \text { (Best Value }=1.0\right)\end{array}$ & $\begin{array}{c}\text { Mean Squared } \\
\text { (Best Value }=\mathbf{0 . 0} \text { ) }\end{array}$ \\
\hline Clinical predictors & 0.54 & 41.87 \\
Clinical predictors + SCCSA & 0.72 & 25.39 \\
Clinical predictors + GMCSA + WMCSA/GMCSA & 0.74 & 23.77 \\
\hline
\end{tabular}

Note:-WMCSA indicates white matter cross-sectional area.

a GMCSA, WMCSA, and SCCSA are averaged across the C4-C6 vertebral levels. Clinical predictors include age, body mass index, sex, site of onset, delay between first symptoms and diagnosis, total ALSFRS-R score at baseline, and total ALSFRS-R score progression rate at baseline.

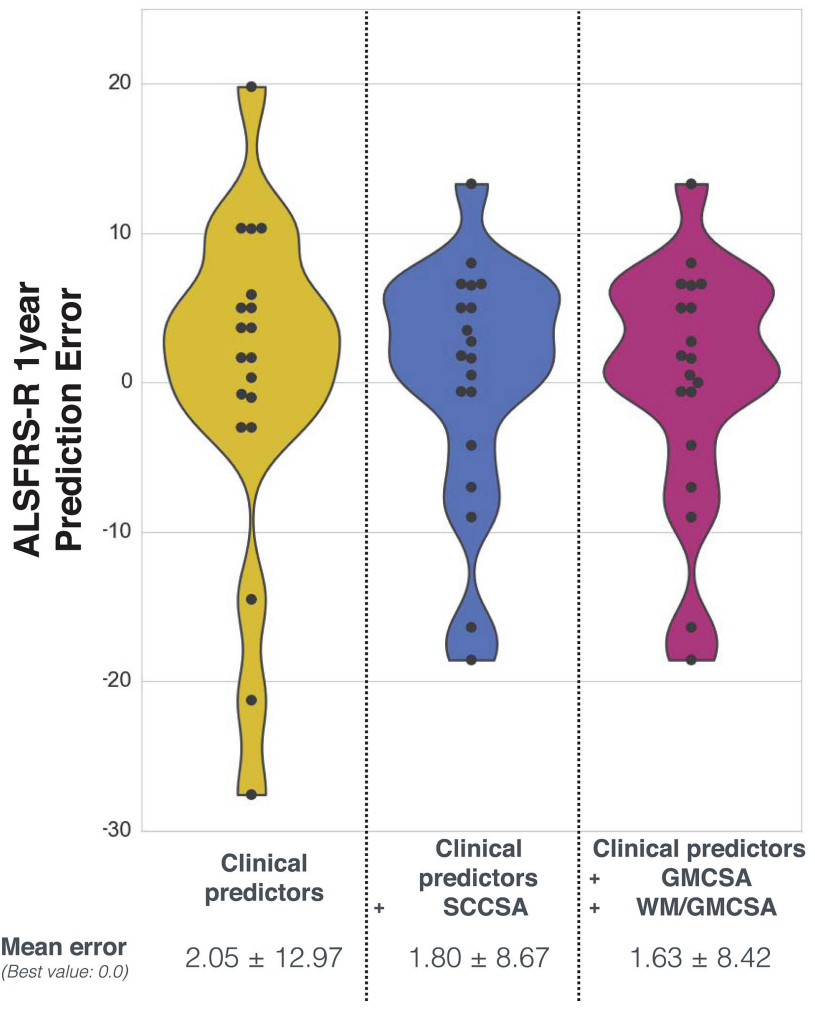

FIG 5. Prediction error on the ALSFRS-R at 1 year, from a leave-oneout cross-validation with regression trees. Results are compared between the regression model including clinical predictors (left distribution plot), clinical predictors + SCCSA (middle distribution plot), and clinical predictors + GMCSA + WM/GMCSA (right distribution plot), where each point represents 1 iteration of the leave-one-out cross-validation. The best value is at 0 .

probable patients with ALS $(n=18)$ and only a few definite patients with ALS $(n=7)$, which may have introduced heterogeneity in the tested ALS population and thus impacted the statistical analyses. However, further analysis performed with the 18 probable patients with ALS led to a correlation between GMCSA and ALSFRS-R of $R=0.61$ and $P=.006$ (versus $R=$ 0.56 and $P=.004$ when including all patients with ALS).

Vertebral Levels. Establishing a vertebral region that maximizes atrophy across patients with ALS was not feasible here because of the large interpatient variability. Consequently, statistical analyses were conducted for each vertebral level between C3-C6. The average CSAs across the C4-C6 vertebral levels was also studied to maximize the sensitivity to clinical markers of the brachial plexus.

In comparison with previous works, ${ }^{3}$ which included levels
C2-T6, the present study only focused on the cervical region from C3-C6 because of the poor quality of the $\mathrm{T} 2{ }^{*}$ weighted images below C6 caused by respiratory-related dynamic B0 field variations. ${ }^{25}$ Thus, the exclusion of the C7 vertebral level slightly restricted the study of the hand muscle deficit. ${ }^{7}$ Moreover, the exclusion of the lumbar and thoracic regions (unavailable in the $\mathrm{T} 2{ }^{*}$ weighted images) precluded studying the correlation between GMCSA and the total ALSFRS-R score, which includes a clinical evaluation of the lower limbs. Optimized MR imaging sequences now provide the possibility to image the thoracic and lumbar GM with satisfactory quality ${ }^{23}$ and should be investigated in future studies.

Measuring CSA with MR Imaging. Being able to measure spinal GM atrophy has potential applications in other diseases, such as spinal muscular atrophy. ${ }^{26}$ Furthermore, an original aspect of the present study is the fully automatic segmentation of the cord and its GM, providing minimum user bias and facilitating the reproducibility of the current technique to other centers (software and processing scripts were made freely available). Previous studies have validated the accuracy of these segmentation methods for the $\mathrm{SC}^{14,27}$ and the $\mathrm{GM},{ }^{8,28}$ including in patients with multiple sclerosis and degenerative cervical myelopathy. ${ }^{28}$ In particular, the GM segmentation used in this study resorts to nonlinear deformations to match the shape of atrophic cord exhibited by patients with ALS. The accuracy has been further validated in the present study in 5 randomly selected patients with ALS and showed satisfactory results (Dice coefficient in the GM $=0.708$ ) in comparison with healthy controls from a previous study ${ }^{8}$ (Dice coefficient in the $\mathrm{GM}=0.711$ ).

Whereas previous studies have reported that the CSA of the SC is associated with morphologic features such as brain volume ${ }^{29}$ and total intracranial volume, ${ }^{30}$ the correlations were mild, and there is no clear consensus as to what is the best normalization method to use ${ }^{31}$; several published studies have not performed CSA normalization, ${ }^{3,26,31-33}$ and neither did we in the present study. Future work could further investigate methods for normalization, and specifically for the GMCSA.

As investigated in a previous study, ${ }^{30}$ age and sex could affect both SC and GMCSA. In the present study, the controls and patients were age-matched but not sex-matched (number of women, 6 with ALS versus 11 controls). However, the larger proportion of women in the control group would in fact decrease the sensitivity to detect a CSA difference between controls and patients, given that both SC and GM are smaller in women compared with men. ${ }^{30}$

Although it would have been relevant to measure CSA in the lateral and anterior funiculi, the current MR imaging protocol, which is based on $\mathrm{T} 2{ }^{*}$-weighted contrast, made it impossible to distinguish the anterior and lateral fasciculus from the rest of the WM tissue. Therefore, it was not feasible to measure specific CSAs of these tracts from our data. Recent work combining multiparametric MR imaging at an ultra-high field showed promising re- 
sults for isolating specific SC tracts, opening the door to such evaluation. $^{34}$

\section{CONCLUSIONS}

Gray matter atrophy as measured noninvasively with MR imaging correlates with clinical disability in ALS at baseline and at 1-year follow-up. Although efforts toward the development of sensitive and reliable biomarkers for ALS need to be pursued and confirmed in larger cohorts, the present study offers an encouraging incentive about the relevance of spinal cord gray matter crosssectional area. This article includes a downloadable link to the MR imaging acquisition and processing protocol to enable other researchers to reproduce the entirety of the analysis performed here. We believe these efforts are critical not only for transparency, but also for standardizing spinal cord imaging biomarkers to help assess their reliability and make them more amenable to clinicians.

\section{ACKNOWLEDGMENTS}

We would like to acknowledge all members of the NeuroPoly Lab, especially Benjamin De Leener and Gabriel Mangeat for the useful discussions, and Dominique Eden for the English review. This study was supported by the Association Française contre les Myopathies-Téléthon (AFM-Téléthon) and the Institut pour la Recherche sur la Moelle épinière et l'Encéphale (IRME). The research leading to these results has also received funding from the program "Investissements d'Avenir" ANR-10-IAIHU-06. We thank the neurologists of the Paris ALS center who participated in the recruitment of the patients in the study: Drs. Nadine Le Forestier, Vincent Meininger, Gaelle Bruneteau; François Salachas, Maria del Mar Amador, and Rabab Debs. We thank Sophie Blancho (IRME) for her help in the management and coordination of the study. We deeply thank the subjects who participated in this research.

Disclosures: Marie-Êve Paquin—RELATED: Grant: Canada Research Chair in Quantitative Magnetic Resonance Imaging (JCA), Canadian Institute of Health Research (CIHR FDN-143263), Canada Foundation for Innovation (32454), Fonds de Recherche du Québec - Santé (28826), Fonds de Recherche du Québec - Nature et Technologies (2015-PR-182754), Natural Sciences and Engineering Research Council of Canada (435897-2013), Quebec Biolmaging Network*. Mohamed M. El Mendili-RELATED: Grant: Association Française contre les Myopathies-Téléthon (AFM-Téléthon), Institut pour la Recherche sur la Moelle épinière et l'Encéphale (IRME), program "Investissements d'Avenir" ANR-10-IAIHU-06, Comments: The funders had no role in study design, data collection and analysis, decision to publish, or preparation of the manuscript*. Charley Gros—RELATED: Grant: Canada Research Chair in Quantitative Magnetic Resonance Imaging (JCA), Canadian Institute of Health Research (CIHR FDN-143263), Canada Foundation for Innovation (32454), Fonds de Recherche du Québec - Santé (28826), Fonds de Recherche du Québec - Nature et Technologies (2015-PR-182754), Natural Sciences and Engineering Research Council of Canada (435897-2013), Quebec Biolmaging Network*. Sara Dupont—RELATED: Grant: Canada Research Chair in Quantitative Magnetic Resonance Imaging (JCA), Canadian Institute of Health Research, (CIHR FDN-143263), Canada Foundation for Innovation (32454), Fonds de Recherche du Québec - Santé (28826), Fonds de Recherche du Québec - Nature et Technologies (2015-PR-182754), Natural Sciences and Engineering Research Council of Canada (435897-2013), Quebec Biolmaging Network*. Julien Cohen-Adad—RELATED: Grant: Canadian Institutes of Health Research*. *Money paid to the institution.

\section{REFERENCES}

1. Chiò A, Logroscino G, Traynor BJ, et al. Global epidemiology of amyotrophic lateral sclerosis: a systematic review of the published literature. Neuroepidemiology 2013;41:118-30 CrossRef Medline
2. Blumenfeld H. Neuroanatomy through Clinical Cases. Sunderland, Massachusetts: Sinauer Associates; 2010

3. El Mendili MM, Cohen-Adad J, Pelegrini-Issac M, et al. Multi-parametric spinal cord MRI as potential progression marker in amyotrophic lateral sclerosis. PLoS One 2014;9:e95516 CrossRef Medline

4. Turner MR, Benatar M. Ensuring continued progress in biomarkers for amyotrophic lateral sclerosis. Muscle Nerve 2015;51:14-18 CrossRef Medline

5. Swinnen B, Robberecht W. The phenotypic variability of amyotrophic lateral sclerosis. Nat Rev Neurol 2014;10:661-70 CrossRef Medline

6. Turner MR, Verstraete E. What does imaging reveal about the pathology of amyotrophic lateral sclerosis? Curr Neurol Neurosci Rep 2015;15:45 CrossRef Medline

7. Cohen-Adad J, El Mendili MM, Morizot-Koutlidis R, et al. Involvement of spinal sensory pathway in ALS and specificity of cord atrophy to lower motor neuron degeneration. Amyotroph Lateral Scler Frontotemporal Degener 2013;14:30-38 CrossRef Medline

8. Dupont SM, De Leener B, Taso M, et al. Fully-integrated framework for the segmentation and registration of the spinal cord white and gray matter. Neuroimage 2017;150:358-72 CrossRef Medline

9. Brooks BR, Miller RG, Swash M, et al. El Escorial revisited: revised criteria for the diagnosis of amyotrophic lateral sclerosis. Amyotroph Lateral Scler Other Motor Neuron Disord 2000;1:293-99 CrossRef Medline

10. Cedarbaum JM, Stambler N, Malta E, et al. The ALSFRS-R: a revised ALS functional rating scale that incorporates assessments of respiratory function. J Neurol Sci 1999;169:13-21 CrossRef Medline

11. De Leener B, Lévy S, Dupont SM, et al. SCT: Spinal Cord Toolbox, an open-source software for processing spinal cord MRI data. Neuroimage 2017;145(Pt A):24-43 CrossRef Medline

12. Fonov VS, Le Troter A, Taso M, et al. Framework for integrated MRI average of the spinal cord white and gray matter: the MNI-PolyAMU template. Neuroimage 2014;102 Pt 2:817-27 CrossRef Medline

13. De Leener B, Fonov V, Collins DL, et al. PAM50: multimodal template of the brainstem and spinal cord compatible with the ICBM152 space. In: Proceedings of the International Society for Magnetic Resonance in Medicine 25th Annual Meeting and Exhibition, Honolulu, Hawaii. April 22-27, 2017

14. De Leener B, Kadoury S, Cohen-Adad J. Robust, accurate and fast automatic segmentation of the spinal cord. Neuroimage 2014;98: 528-36 CrossRef Medline

15. De Leener B, Taso M, Fonov V, et al. Fully-integrated T1, T2, T2*, white and gray matter atlases of the spinal cord. In: Proceedings of the International Society for Magnetic Resonance in Medicine 24th Annual Meeting and Exhibition, Singapore. May 7-8, 2016

16. Tustison NJ, Avants BB. Explicit B-spline regularization in diffeomorphic image registration. Front Neuroinform 2013;7:39 CrossRef Medline

17. Avants BB, Epstein CL, Grossman M, et al. Symmetric diffeomorphic image registration with cross-correlation: evaluating automated labeling of elderly and neurodegenerative brain. Med Image Anal 2008;12:26-41 CrossRef Medline

18. Avants BB, Tustison NJ, Stauffer M, et al. The Insight ToolKit image registration framework. Front Neuroinform 2014;8:44 CrossRef Medline

19. Cohen-Adad J, Levy S, Avants B. Slice-by-slice regularized registration for spinal cord MRI: SliceReg. In: Proceedings of the International Society for Magnetic Resonance in Medicine 23rd Annual Meeting and Exhibition, Toronto, Canada. May 30-June 5, 2015

20. Breiman L, Friedman JH, Olshen RA, et al. Classification and Regression Trees. Belmont, California: Wadsworth International Group; 1984

21. Kushner PD, Stephenson DT, Wright S. Reactive astrogliosis is widespread in the subcortical white matter of amyotrophic lateral sclerosis brain. J Neuropathol Exp Neurol 1991;50:263-77 CrossRef Medline

22. Sitte HH, Wanschitz J, Budka H, et al. Autoradiography with [3 H] 
PK11195 of spinal tract degeneration in amyotrophic lateral sclerosis. Acta Neuropathol 2001;101:75-78 Medline

23. Yiannakas MC, Kakar P, Hoy LR, et al. The use of the lumbosacral enlargement as an intrinsic imaging biomarker: feasibility of grey matter and white matter cross-sectional area measurements using MRI at 3T. PLoS One 2014;9:e105544 CrossRef Medline

24. van der Burgh HK, Schmidt R, Westeneng HJ, et al. Deep learning predictions of survival based on MRI in amyotrophic lateral sclerosis. Neuroimage Clin 2017;13:361-69 CrossRef Medline

25. Verma T, Cohen-Adad J. Effect of respiration on the B0 field in the human spinal cord at 3T. Magn Reson Med 2014;72:1629-36 CrossRef Medline

26. El Mendili MM, Lenglet T, Stojkovic T, et al. Cervical spinal cord atrophy profile in adult SMN1-linked SMA. PLoS One 2016;11: e0152439 CrossRef Medline

27. Yiannakas MC, Mustafa AM, De Leener B, et al. Fully automated segmentation of the cervical cord from T1-weighted MRI using PropSeg: application to multiple sclerosis. Neuroimage Clin 2016; 10:71-77 CrossRef Medline

28. Dupont SM, Martin AR, Stikov N, et al. Fully automatic segmentation of spinal cord gray matter on patients with degenerative cervical myelopathy. In: Proceedings of the International Society for Magnetic Resonance in Medicine 25th Annual Meeting and Exhibition. Honolulu, Hawaii; April 22-27, 2017

29. Engl C, Schmidt P, Arsic M, et al. Brain size and white matter content of cerebrospinal tracts determine the upper cervical cord area: evidence from structural brain MRI. Neuroradiology 2013;55:963-70 CrossRef Medline

30. Papinutto N, Schlaeger R, Panara V, et al. Age, gender and normalization covariates for spinal cord gray matter and total cross-sectional areas at cervical and thoracic levels: a $2 \mathrm{D}$ phase sensitive inversion recovery imaging study. PLoS One 2015;10:e118576 CrossRef Medline

31. Healy BC, Arora A, Hayden DL, et al. Approaches to normalization of spinal cord volume: application to multiple sclerosis. J Neuroimaging 2012;22:e12-e19 CrossRef Medline

32. Behmanesh B, Gessler F, Quick-Weller J, et al. Regional spinal cord atrophy is associated with poor outcome after surgery on intramedullary spinal cord ependymoma: a new aspect of delayed neurological deterioration. World Neurosurg 2017;100:250-55 CrossRef Medline

33. Kearney H, Yiannakas MC, Abdel-Aziz K, et al. Improved MRI quantification of spinal cord atrophy in multiple sclerosis. J Magn Reson Imaging 2014;39:617-23 CrossRef Medline

34. Taso M, Massire A, Besson P, et al. Towards in vivo spinal cord cytoand myelo-architecture deciphering using multi-modal MRI parcellation at 7T. In: Proceedings of the International Society for Magnetic Resonance in Medicine 25th Annual Meeting and Exhibition, Honolulu, Hawaii. April 22-27, 2017 